\title{
Commentary: Checklist fatigue? A unique opportunity in cardiac surgical care. The impact of a voluntary, cardiac surgery-specific safety checklist
}

\author{
Juan N. Pulido, MD
}

\footnotetext{
From Cardiovascular Intensive Care Unit, Cardiothoracic Anesthesiology and Critical Care Medicine, Swedish Heart and Vascular Institute, Swedish Medical Center, US Anesthesia Partners Washington, Seattle, Wash. Disclosures: Author has nothing to disclose with regard to commercial support.

Received for publication Aug 29, 2019; accepted for publication Aug 29, 2019; available ahead of print Oct 11, 2019.

Address for reprints: Juan N. Pulido, MD, Cardiovascular Intensive Care Unit, Cardiothoracic Anesthesiology and Critical Care Medicine, Swedish Heart and Vascular Institute, Swedish Medical Center, US Anesthesia Partners Washington, 550 17th Ave \#680, Seattle, WA 98122 (E-mail: juan.pulido@ swedish.org).

J Thorac Cardiovasc Surg 2020;159:1891-2

$0022-5223 / \$ 36.00$

Copyright (C) 2019 by The American Association for Thoracic Surgery

https://doi.org/10.1016/j.jtcvs.2019.08.115
}

Surgical safety checklists are now engrained in our daily lives and considered a standard in perioperative care. It has been over a decade since the World Health Organization developed the first edition of the 19-point checklist that was shown to reduce morbidity and mortality in patients undergoing noncardiac surgery in a diverse group of hospitals around the world. ${ }^{1}$ Since that time, adoption and implementation of checklists, using the aviation industry as an example, have become not only a standard but an obligation and have flooded the entire health care spectrum with significant emphasis in procedural areas of care. The most recent and largest meta-analysis ${ }^{2}$ on this subject included more than 44,000 patients from 497 hospitals in 27 countries and demonstrated that patients exposed to surgical safety checklists experience better postoperative outcomes.

Although the impact of safety checklist implementation is clear, the essential inherent benefit comes from providing a specific tool to engage a team and ensure minimal standards are met, avoid ambiguity, and encourage participation of all team members. Unfortunately, the real meaningful use has occasionally been tainted by "checklist fatigue" and "just checking a box" behavior, as we add more and more checklists to the daily routine to safely navigate with the patient through the multiple stages of perioperative care. Despite these challenges, checklists are here to stay.

In this issue of the Journal, Spanjersberg and colleagues ${ }^{3}$ evaluate the addition of a voluntary, specific pre-incision safety checklist in cardiac surgical patients in a large prospective, observational, multicenter cohort study. The study was performed in the Netherlands and evaluated close to 6000 consecutive patients over a period of 1 year. To be clear, all hospitals already had a standard surgical time out of 11 safety checklist items that was an expectation in all centers since 2009. This was an additional 5-step cardiac

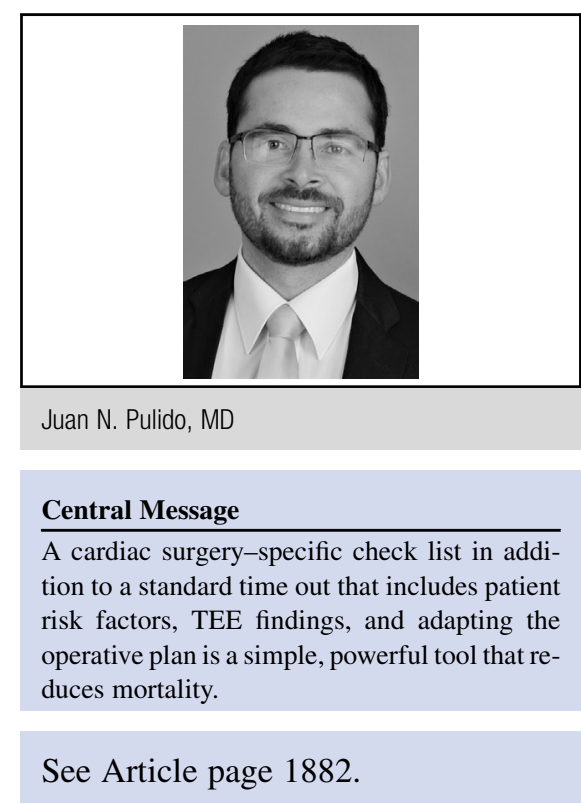

surgery-specific checklist that provided individual patient risk factors and incorporated transesophageal echocardiography (TEE) findings with the option of an adapt procedure or change of plan.

Some key aspects of this initiative included the fact that it was voluntary and specific to the particular patient at hand. The centers had the option to opt out. This feature ensured team engagement, fomented discussion and communication, minimized the "just clicking the box" behavior, and maximized the power of the checklist. Moreover, it incorporated specific pre-incision TEE findings with chance of operative modification or adapting the procedure, such as change in cannulation strategy, clamping location, or insertion of intra-aortic balloon pump. As the adherence to the pre-incision-specific checklist was voluntary, there was expected variability in each institution per month, with a progressive increase in implementation by the end of the study. As a result from its use, $16 \%$ of patients had adaptations to the original surgical plan. More importantly, the 30-day and 120-day mortality was reduced with the use of the added specific checklist. This effect persisted after correction for age, sex, left ventricular function, renal function, participating hospital, emergency surgery, and type of surgery.

The study has some inherent limitations that the authors describe eloquently. It's important to highlight that outcome 
data are usually dependent on reporting from participating institutions, and as complications tend to be underreported, there is always the possibility of selection bias.

Regardless of the limitations, this is a great example how a simple intervention that engages the team and foments a collaborative approach to patient care has had an impact in mortality. Moreover, cardiac surgery provides a unique opportunity for this initiative, as TEE findings can occasionally change the plan.

\section{References}

1. Haynes AB, Weiser TG, Berry WR, Lipsitz SR, Breizat AH, Dellinger EP, et al. A surgical safety checklist to reduce morbidity and mortality in a global population. N Engl J Med. 2009;360:491-9.

2. Abbott TEF, Ahmad T, Phull MK, Fowler AJ, Hewson R, Biccard BM, et al. The surgical safety checklist and patient outcomes after surgery: a prospective observational cohort study, systematic review and meta-analysis. $\mathrm{Br} J$ Anaesth. 2018;120:146e155.

3. Spanjersberg AJ, Ottervanger JP, Nierich AP, Speekenbrink RGH, Stooker W, Hoogendoorn $\mathrm{M}$, et al. Implementation of a specific safety check is associated with lower postoperative mortality in cardiac surgery. J Thorac Cardiovasc Surg. 2020;159:1882-90.e2. 